\title{
Automatic Text Scoring Using Neural Networks
}

\author{
Dimitrios Alikaniotis \\ Department of Theoretical \\ and Applied Linguistics \\ University of Cambridge \\ Cambridge, UK \\ da352@cam.ac.uk
}

\author{
Helen Yannakoudakis \\ The ALTA Institute \\ Computer Laboratory \\ University of Cambridge \\ Cambridge, UK \\ hy260@cl.cam.ac.uk
}

\author{
Marek Rei \\ The ALTA Institute \\ Computer Laboratory \\ University of Cambridge \\ Cambridge, UK \\ mr472@cl.cam.ac.uk
}

\begin{abstract}
Automated Text Scoring (ATS) provides a cost-effective and consistent alternative to human marking. However, in order to achieve good performance, the predictive features of the system need to be manually engineered by human experts. We introduce a model that forms word representations by learning the extent to which specific words contribute to the text's score. Using Long-Short Term Memory networks to represent the meaning of texts, we demonstrate that a fully automated framework is able to achieve excellent results over similar approaches. In an attempt to make our results more interpretable, and inspired by recent advances in visualizing neural networks, we introduce a novel method for identifying the regions of the text that the model has found more discriminative.
\end{abstract}

\section{Introduction}

Automated Text Scoring (ATS) refers to the set of statistical and natural language processing techniques used to automatically score a text on a marking scale. The advantages of ATS systems have been established since Project Essay Grade (PEG) (Page, 1967; Page, 1968), one of the earliest systems whose development was largely motivated by the prospect of reducing labour-intensive marking activities. In addition to providing a cost-effective and efficient approach to large-scale grading of (extended) text, such systems ensure a consistent application of marking criteria, therefore facilitating equity in scoring.

There is a large body of literature with regards to ATS systems of text produced by nonnative English-language learners (Page, 1968; At- tali and Burstein, 2006; Rudner and Liang, 2002; Elliot, 2003; Landauer et al., 2003; Briscoe et al., 2010; Yannakoudakis et al., 2011; Sakaguchi et al., 2015, among others), overviews of which can be found in various studies (Williamson, 2009; Dikli, 2006; Shermis and Hammer, 2012). Implicitly or explicitly, previous work has primarily treated text scoring as a supervised text classification task, and has utilized a large selection of techniques, ranging from the use of syntactic parsers, via vectorial semantics combined with dimensionality reduction, to generative and discriminative machine learning.

As multiple factors influence the quality of texts, ATS systems typically exploit a large range of textual features that correspond to different properties of text, such as grammar, vocabulary, style, topic relevance, and discourse coherence and cohesion. In addition to lexical and part-ofspeech (POS) $n$ grams, linguistically deeper features such as types of syntactic constructions, grammatical relations and measures of sentence complexity are among some of the properties that form an ATS system's internal marking criteria. The final representation of a text typically consists of a vector of features that have been manually selected and tuned to predict a score on a marking scale.

Although current approaches to scoring, such as regression and ranking, have been shown to achieve performance that is indistinguishable from that of human examiners, there is substantial manual effort involved in reaching these results on different domains, genres, prompts and so forth. Linguistic features intended to capture the aspects of writing to be assessed are hand-selected and tuned for specific domains. In order to perform well on different data, separate models with distinct feature sets are typically tuned. 
Prompted by recent advances in deep learning and the ability of such systems to surpass state-ofthe-art models in similar areas (Tang, 2015; Tai et al., 2015), we propose the use of recurrent neural network models for ATS. Multi-layer neural networks are known for automatically learning useful features from data, with lower layers learning basic feature detectors and upper levels learning more high-level abstract features (Lee et al., 2009). Additionally, recurrent neural networks are well-suited for modeling the compositionality of language and have been shown to perform very well on the task of language modeling (Mikolov et al., 2011; Chelba et al., 2013). We therefore propose to apply these network structures to the task of scoring, in order to both improve the performance of ATS systems and learn the required feature representations for each dataset automatically, without the need for manual tuning. More specifically, we focus on predicting a holistic score for extended-response writing items. ${ }^{1}$

However, automated models are not a panacea, and their deployment depends largely on the ability to examine their characteristics, whether they measure what is intended to be measured, and whether their internal marking criteria can be interpreted in a meaningful and useful way. The deep architecture of neural network models, however, makes it rather difficult to identify and extract those properties of text that the network has identified as discriminative. Therefore, we also describe a preliminary method for visualizing the information the model is exploiting when assigning a specific score to an input text.

\section{Related Work}

In this section, we describe a number of the more influential and/or recent approaches in automated text scoring of non-native English-learner writing.

Project Essay Grade (Page, 1967; Page, 1968; Page, 2003) is one of the earliest automated scoring systems, predicting a score using linear regression over vectors of textual features considered to be proxies of writing quality. Intelligent Essay Assessor (Landauer et al., 2003) uses Latent Semantic Analysis to compute the semantic similarity between texts at specific grade points and a test text, which is assigned a score based on the ones in

\footnotetext{
${ }^{1}$ The task is also referred to as Automated Essay Scoring. Throughout this paper, we use the terms text and essay (scoring) interchangeably.
}

the training set to which it is most similar. Lonsdale and Strong-Krause (2003) use the Link Grammar parser (Sleator and Templerley, 1995) to analyse and score texts based on the average sentencelevel scores calculated from the parser's cost vector.

The Bayesian Essay Test Scoring sYstem (Rudner and Liang, 2002) investigates multinomial and Bernoulli Naive Bayes models to classify texts based on shallow content and style features. eRater (Attali and Burstein, 2006), developed by the Educational Testing Service, was one of the first systems to be deployed for operational scoring in high-stakes assessments. The model uses a number of different features, including aspects of grammar, vocabulary and style (among others), whose weights are fitted to a marking scheme by regression.

Chen et al. (2010) use a voting algorithm and address text scoring within a weakly supervised bag-of-words framework. Yannakoudakis et al. (2011) extract deep linguistic features and employ a discriminative learning-to-rank model that outperforms regression.

Recently, McNamara et al. (2015) used a hierachical classification approach to scoring, utilizing linguistic, semantic and rhetorical features, among others. Farra et al. (2015) utilize variants of logistic and linear regression and develop models that score persuasive essays based on features extracted from opinion expressions and topical elements.

There have also been attempts to incorporate more diverse features to text scoring models. Klebanov and Flor (2013) demonstrate that essay scoring performance is improved by adding to the model information about percentages of highly associated, mildly associated and dis-associated pairs of words that co-exist in a given text. Somasundaran et al. (2014) exploit lexical chains and their interaction with discourse elements for evaluating the quality of persuasive essays with respect to discourse coherence. Crossley et al. (2015) identify student attributes, such as standardized test scores, as predictive of writing success and use them in conjunction with textual features to develop essay scoring models.

In 2012, Kaggle, ${ }^{2}$ sponsored by the Hewlett Foundation, hosted the Automated Student Assessment Prize (ASAP) contest, aiming to demon-

\footnotetext{
${ }^{2}$ http://www.kaggle.com/c/asap-aes/
} 
strate the capabilities of automated text scoring systems (Shermis, 2015). The dataset released consists of around twenty thousand texts $(60 \%$ of which are marked), produced by middle-school English-speaking students, which we use as part of our experiments to develop our models.

\section{Models}

\subsection{C\&W Embeddings}

Collobert and Weston (2008) and Collobert et al. (2011) introduce a neural network architecture (Fig. 1a) that learns a distributed representation for each word $w$ in a corpus based on its local context. Concretely, suppose we want to learn a representation for some target word $w_{t}$ found in an $n$-sized sequence of words $\mathcal{S}=\left(w_{1}, \ldots, w_{t}, \ldots, w_{n}\right)$ based on the other words which exist in the same sequence $\left(\forall w_{i} \in \mathcal{S} \mid w_{i} \neq w_{t}\right)$. In order to derive this representation, the model learns to discriminate between $\mathcal{S}$ and some 'noisy' counterpart $\mathcal{S}^{\prime}$ in which the target word $w_{t}$ has been substituted for a randomly sampled word from the vocabulary: $\mathcal{S}^{\prime}=\left(w_{1}, \ldots, w_{c}, \ldots, w_{n} \mid w_{c} \sim \mathcal{V}\right)$. In this way, every word $w$ is more predictive of its local context than any other random word in the corpus.

Every word in $\mathcal{V}$ is mapped to a real-valued vector in $\Omega$ via a mapping function $C(\cdot)$ such that $C\left(w_{i}\right)=\left\langle\mathbf{M}_{\star i}\right\rangle$, where $\mathbf{M} \in \mathbb{R}^{D \times|\mathcal{V}|}$ is the embedding matrix and $\left\langle\mathbf{M}_{\star i}\right\rangle$ is the $i$ th column of $\mathbf{M}$. The network takes $\mathcal{S}$ as input by concatenating the vectors of the words found in it; $\mathbf{s}_{t}=\left\langle C\left(w_{1}\right)^{\top}\|\ldots\| C\left(w_{t}\right)^{\top}\|\ldots\| C\left(w_{n}\right)^{\top}\right\rangle \in$ $\mathbb{R}^{n D}$. Similarly, $\mathcal{S}^{\prime}$ is formed by substituting $C\left(w_{t}\right)$ for $C\left(w_{c}\right) \sim \mathbf{M} \mid w_{c} \neq w_{t}$.

The input vector is then passed through a hard tanh layer defined as,

$$
h \tanh (x)= \begin{cases}-1 & x<-1 \\ x & -1 \leqslant x \leqslant 1 \\ 1 & x>1\end{cases}
$$

which feeds a single linear unit in the output layer. The function that is computed by the network is ultimately given by (4):

$$
\begin{aligned}
\mathbf{s}_{t} & =\left\langle\mathbf{M}_{\star 1}^{\top}\|\ldots\| \mathbf{M}_{\star t}^{\top}\|\ldots\| \mathbf{M}_{\star n}^{\top}\right\rangle^{\top} \\
\mathbf{i} & =\sigma\left(\mathbf{W}_{h i} \mathbf{s}_{t}+\mathbf{b}_{h}\right) \\
f\left(\mathbf{s}_{t}\right) & =\mathbf{W}_{o h} \mathbf{i}+\mathbf{b}_{o}
\end{aligned}
$$

$$
\begin{aligned}
f(\mathbf{s}), \mathbf{b}_{o} & \in \mathbb{R}^{1} \\
\mathbf{W}_{o h} & \in \mathbb{R}^{H \times 1} \\
\mathbf{W}_{h i} & \in \mathbb{R}^{D \times H} \\
\mathbf{s} & \in \mathbb{R}^{D} \\
\mathbf{b}_{o} & \in \mathbb{R}^{H}
\end{aligned}
$$

where $\mathbf{M}, \mathbf{W}_{o h}, \mathbf{W}_{h i}, \mathbf{b}_{o}, \mathbf{b}_{h}$ are learnable parameters, $D, H$ are hyperparameters controlling the size of the input and the hidden layer, respectively; $\sigma$ is the application of an element-wise non-linear function (htanh in this case).

The model learns word embeddings by ranking the activation of the true sequence $\mathcal{S}$ higher than the activation of its 'noisy' counterpart $\mathcal{S}^{\prime}$. The objective of the model then becomes to minimize the hinge loss which ensures that the activations of the original and 'noisy' $n$ grams will differ by at least 1:

$$
\begin{aligned}
& \text { loss }_{\text {context }}(\text { target }, \text { corrupt })= \\
& \quad\left[1-f\left(\mathbf{s}_{t}\right)+f\left(\mathbf{s}_{c k}\right)\right]_{+}, \forall k \in \mathbb{Z}^{E}
\end{aligned}
$$

where $E$ is another hyperparameter controlling the number of 'noisy' sequences we give along with the correct sequence (Mikolov et al., 2013; Gutmann and Hyvärinen, 2012).

\subsection{Augmented C\&W model}

Following Tang (2015), we extend the previous model to capture not only the local linguistic environment of each word, but also how each word contributes to the overall score of the essay. The aim here is to construct representations which, along with the linguistic information given by the linear order of the words in each sentence, are able to capture usage information. Words such as is, are, to, at which appear with any essay score are considered to be under-informative in the sense that they will activate equally both on high and low scoring essays. Informative words, on the other hand, are the ones which would have an impact on the essay score (e.g., spelling mistakes).

In order to capture those score-specific word embeddings (SSWES), we extend (4) by adding a further linear unit in the output layer that performs linear regression, predicting the essay score. Using (2), the activations of the network (presented in Fig. 1b) are given by: 


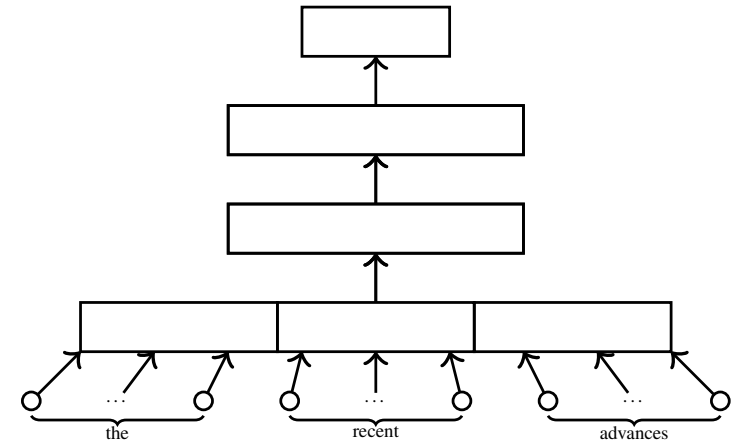

(a)

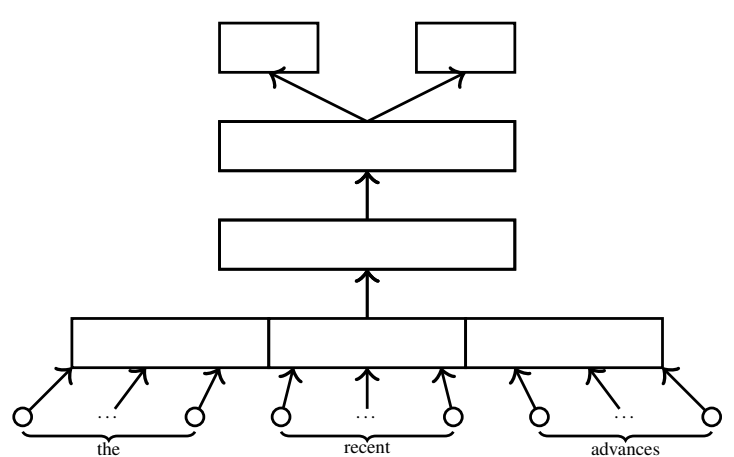

(b)

Figure 1: Architecture of the original $\mathrm{C} \& \mathrm{~W}$ model (left) and of our extended version (right).

$$
\begin{aligned}
f_{\text {ss }}(\mathbf{s})=\mathbf{W}_{\text {oh }_{1}} \mathbf{i}+\mathbf{b}_{o_{1}} \\
f_{\text {context }}(\mathbf{s})=\mathbf{W}_{\text {oh }_{2}} \mathbf{i}+\mathbf{b}_{o_{2}} \\
f_{\text {ss }}(\mathbf{s}) \in[\min (\text { score }), \max (\text { score })] \\
\mathbf{b}_{o_{1}} \in \mathbb{R}^{1} \\
\mathbf{W}_{\text {oh }_{1}} \in \mathbb{R}^{1 \times H}
\end{aligned}
$$

The error we minimize for $f_{s s}$ (where $s s$ stands for score specific) is the mean squared error between the predicted $\hat{y}$ and the actual essay score $y$ :

$$
\operatorname{loss}_{\text {score }}(\mathbf{s})=\frac{1}{N} \sum_{i=1}^{N}\left(\hat{y}_{i}-y_{i}\right)^{2}
$$

From (5) and (8) we compute the overall loss function as a weighted linear combination of the two loss functions (9), back-propagating the error gradients to the embedding matrix $\mathbf{M}$ :

$$
\operatorname{loss}_{\text {overall }}(\mathbf{s})=\begin{aligned}
& \alpha \cdot \operatorname{loss}_{\text {context }}\left(\mathbf{s}, \mathbf{s}^{\prime}\right) \\
& +(1-\alpha) \cdot \operatorname{loss}_{\text {score }}(\mathbf{s})
\end{aligned}
$$

where $\alpha$ is the hyper-parameter determining how the two error functions should be weighted. $\alpha$ values closer to 0 will place more weight on the scorespecific aspect of the embeddings, whereas values closer to 1 will favour the contextual information.

Fig. 2 shows the advantage of using SSWEs in the present setting. Based solely on the information provided by the linguistic environment, words such as computer and laptop are going to be placed together with their mis-spelled counterparts copmuter and labtop (Fig. 2a). This, however, does not reflect the fact that the mis-spelled words tend to appear in lower scoring essays. Using SSWEs, the correctly spelled words are pulled apart in the vector space from the incorrectly spelled ones, retaining, however, the information that labtop and copmuter are still contextually related (Fig. 2b).

\subsection{Long-Short Term Memory Network}

We use the SSWES obtained by our model to derive continuous representations for each essay. We treat each essay as a sequence of tokens and explore the use of uni- and bi-directional (Graves, 2012) Long-Short Term Memory networks (LSTMs) (Hochreiter and Schmidhuber, 1997) in order to embed these sequences in a vector of fixed size. Both uni- and bi-directional LSTMs have been effectively used for embedding long sequences (Hermann et al., 2015). LSTMs are a kind of recurrent neural network (RNN) architecture in which the output at time $t$ is conditioned on the input $\mathbf{s}$ both at time $t$ and at time $t-1$ :

$$
\begin{aligned}
\mathbf{y}_{t} & =\mathbf{W}_{y h} \mathbf{h}_{t}+\mathbf{b}_{y} \\
\mathbf{h}_{t} & =\mathcal{H}\left(\mathbf{W}_{h s} \mathbf{s}_{t}+\mathbf{W}_{h h} \mathbf{h}_{t-1}+\mathbf{b}_{h}\right)
\end{aligned}
$$

where $\mathbf{s}_{t}$ is the input at time $t$, and $\mathcal{H}$ is usually an element-wise application of a non-linear function. In LSTMs, $\mathcal{H}$ is substituted for a composite function defining $\mathbf{h}_{t}$ as:

$$
\begin{gathered}
\mathbf{i}_{t}=\begin{array}{c}
\sigma\left(\mathbf{W}_{i s} \mathbf{s}_{t}+\mathbf{W}_{i h} \mathbf{h}_{t-1}+\right. \\
\left.\mathbf{W}_{i c} \mathbf{c}_{t-1}+\mathbf{b}_{i}\right)
\end{array} \\
\mathbf{f}_{t}=\begin{array}{c}
\sigma\left(\mathbf{W}_{f s} \mathbf{s}_{t}+\mathbf{W}_{f h} \mathbf{h}_{t-1}+\right. \\
\left.\mathbf{W}_{f c} \mathbf{c}_{t-1}+\mathbf{b}_{f}\right)
\end{array} \\
\mathbf{c}_{t}=\begin{array}{c}
\mathbf{i}_{t} \odot g\left(\mathbf{W}_{c s} \mathbf{s}_{t}+\mathbf{W}_{c h} \mathbf{h}_{t-1}+\mathbf{b}_{c}\right)+ \\
\mathbf{f}_{t} \odot \mathbf{c}_{t-1}
\end{array}
\end{gathered}
$$




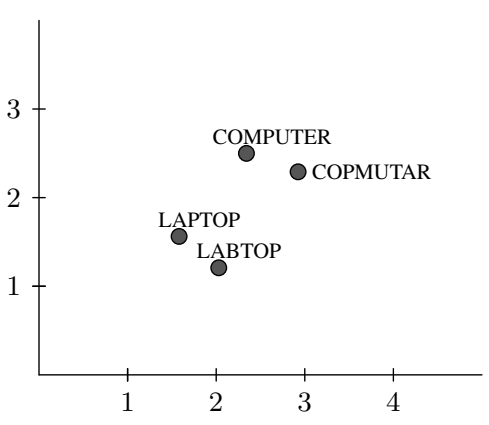

(a)

Standard neural embeddings

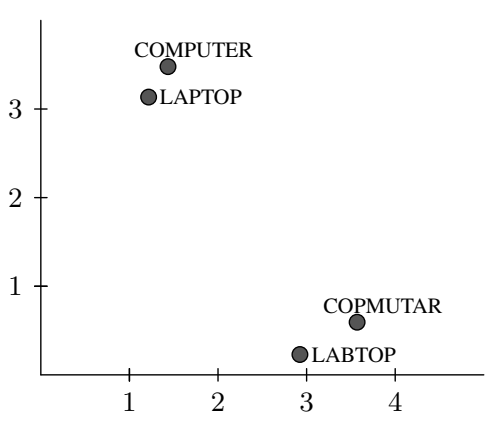

(b)

Score-specific word embeddings

Figure 2: Comparison between standard and score-specific word embeddings. By virtue of appearing in similar environments, standard neural embeddings will place the correct and the incorrect spelling closer in the vector space. However, since the mistakes are found in lower scoring essays, SSWEs are able to discriminate between the correct and the incorrect versions without loss in contextual meaning.

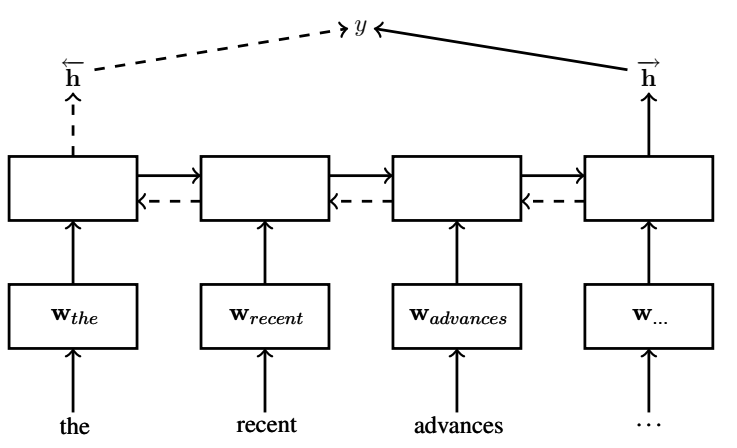

Figure 3: A single-layer Long Short Term Memory (LSTM) network. The word vectors $\mathbf{w}_{i}$ enter the input layer one at a time. The hidden layer that has been formed at the last timestep is used to predict the essay score using linear regression. We also explore the use of bi-directional LSTMs (dashed arrows). For 'deeper' representations, we can stack more LSTM layers after the hidden layer shown here.

$$
\begin{aligned}
\mathbf{o}_{t} & =\begin{array}{r}
\sigma\left(\mathbf{W}_{o s} \mathbf{s}_{t}+\mathbf{W}_{o h} \mathbf{h}_{t-1}+\right. \\
\left.\mathbf{W}_{o c} \mathbf{c}_{t}+\mathbf{b}_{o}\right)
\end{array} \\
\mathbf{h}_{t} & =\mathbf{o}_{t} \odot h\left(\mathbf{c}_{t}\right)
\end{aligned}
$$

where $g, \sigma$ and $h$ are element-wise non-linear functions such as the logistic sigmoid $\left(\frac{1}{1+e^{-x}}\right)$ and the hyperbolic tangent $\left(\frac{e^{2 z}-1}{e^{2 z}+1}\right) ; \odot$ is the Hadamard product; $\mathbf{W}, \mathbf{b}$ are the learned weights and biases respectively; and $i, f, o$ and $c$ are the input, forget, output gates and the cell activation vectors respectively.

Training the LSTM in a uni-directional manner (i.e., from left to right) might leave out important information about the sentence. For example, our interpretation of a word at some point $t_{i}$ might be different once we know the word at $t_{i+5}$. An effective way to get around this issue has been to train the LSTM in a bidirectional manner. This requires doing both a forward and a backward pass of the sequence (i.e., feeding the words from left to right and from right to left). The hidden layer element in (10) can therefore be re-written as the concatenation of the forward and backward hidden vectors:

$$
\mathbf{y}_{t}=\mathbf{W}_{y h}\left(\begin{array}{c}
\overleftarrow{\mathbf{h}}_{t}^{\top} \\
\overrightarrow{\mathbf{h}}_{t}^{\top}
\end{array}\right)+\mathbf{b}_{y}
$$

We feed the embedding of each word found in each essay to the LSTM one at a time, zero-padding shorter sequences. We form $D$ dimensional essay embeddings by taking the activation of the LSTM layer at the timestep where the last word of the essay was presented to the network. In the case of bi-directional LSTMs, the two independent passes of the essay (from left to right and from right to left) are concatenated together to predict the essay score. These essay embeddings are then fed to a linear unit in the output layer which predicts the essay score (Fig. 3). We use the mean square error between the predicted and the gold score as our loss function, and optimize with RMSprop (Dauphin et al., 2015), propagating the errors back to the word embeddings. ${ }^{3}$

\footnotetext{
${ }^{3}$ The maximum time for jointly training a particular SSWE + LSTM combination took about 55-60 hours on an Amazon EC2 g2.2xlarge instance (average time was 27-30 hours).
} 


\subsection{Other Baselines}

We train a Support Vector Regression model (see Section 4), which is one of the most widely used approaches in text scoring. We parse the data using the RASP parser (Briscoe et al., 2006) and extract a number of different features for assessing the quality of the essays. More specifically, we use character and part-of-speech unigrams, bigrams and trigrams; word unigrams, bigrams and trigrams where we replace open-class words with their POS; and the distribution of common nouns, prepositions, and coordinators. Additionally, we extract and use as features the rules from the phrase-structure tree based on the top parse for each sentence, as well as an estimate of the error rate based on manually-derived error rules.

$N$ grams are weighted using $t f-i d f$, while the rest are count-based and scaled so that all features have approximately the same order of magnitude. The final input vectors are unit-normalized to account for varying text-length biases.

Further to the above, we also explore the use of the Distributed Memory Model of Paragraph Vectors (PV-DM) proposed by Le and Mikolov (2014), as a means to directly obtain essay embeddings. PV-DM takes as input word vectors which make up $n$ gram sequences and uses those to predict the next word in the sequence. A feature of PV-DM, however, is that each 'paragraph' is assigned a unique vector which is used in the prediction. This vector, therefore, acts as a 'memory', retaining information from all contexts that have appeared in this paragraph. Paragraph vectors are then fed to a linear regression model to obtain essay scores (we refer to this model as doc $2 \mathrm{vec}$ ).

Additionally, we explore the effect of our scorespecific method for learning word embeddings, when compared against three different kinds of word embeddings:

- word2vec embeddings (Mikolov et al., 2013) trained on our training set (see Section 4).

- Publicly available word2vec embeddings (Mikolov et al., 2013) pre-trained on the Google News corpus (ca. 100 billion words), which have been very effective in capturing solely contextual information.

- Embeddings that are constructed on the fly by the LSTM, by propagating the errors from its hidden layer back to the embedding matrix (i.e., we do not provide any pre-trained word embeddings). ${ }^{4}$

\section{Dataset}

The Kaggle dataset contains 12.976 essays ranging from 150 to 550 words each, marked by two raters (Cohen's $\kappa=0.86$ ). The essays were written by students ranging from Grade 7 to Grade 10, comprising eight distinct sets elicited by eight different prompts, each with distinct marking criteria and score range. ${ }^{5}$ For our experiments, we use the resolved combined score between the two raters, which is calculated as the average between the two raters' scores (if the scores are close), or is determined by a third expert (if the scores are far apart). Currently, the state-of-the-art on this dataset has achieved a Cohen's $\kappa=0.81$ (using quadratic weights). However, the test set was released without the gold score annotations, rendering any comparisons futile, and we are therefore restricted in splitting the given training set to create a new test set.

The sets where divided as follows: $80 \%$ of the entire dataset was reserved for training/validation, and $20 \%$ for testing. $80 \%$ of the training/validation subset was used for actual training, while the remaining $20 \%$ for validation (in absolute terms for the entire dataset: $64 \%$ training, $16 \%$ validation, $20 \%$ testing). To facilitate future work, we release the ids of the validation and test set essays we used in our experiments, in addition to our source code and various hyperparameter values. ${ }^{6}$

\section{Experiments}

\subsection{Results}

The hyperparameters for our model were as follows: sizes of the layers $H, D$, the learning rate $\eta$, the window size $n$, the number of 'noisy' sequences $E$ and the weighting factor $\alpha$. Also the hyperparameters of the LSTM were the size of the LSTM layer $D_{L S T M}$ as well as the dropout rate $r$.

\footnotetext{
${ }^{4}$ Another option would be to use standard C\&W embeddings; however, this is equivalent to using SSWEs with $\alpha=1$, which we found to produce low results.

${ }^{5}$ Five prompts employed a holistic scoring rubric, one was scored with a two-trait rubric, and two were scored with a multi-trait rubric, but reported as a holistic score (Shermis and Hammer, 2012).

${ }^{6}$ The code, by-model hyperparameter configurations and the IDs of the testing set are available at https:// github.com/dimalik/ats/.
} 


\begin{tabular}{|l|c|c|c|c|}
\hline Model & Spearman's $\rho$ & Pearson $r$ & RMSE & Cohen's $\kappa$ \\
\hline doc2vec & 0.62 & 0.63 & 4.43 & 0.85 \\
\hline SVM & 0.78 & 0.77 & 8.85 & 0.75 \\
\hline LSTM & 0.59 & 0.60 & 6.8 & 0.54 \\
\hline BLSTM & 0.7 & 0.5 & 7.32 & 0.36 \\
\hline Two-layer LSTM & 0.58 & 0.55 & 7.16 & 0.46 \\
\hline Two-layer BLSTM & 0.68 & 0.52 & 7.31 & 0.48 \\
\hline word2vec + LSTM & 0.68 & 0.77 & 5.39 & 0.76 \\
\hline word2vec + BLSTM & 0.75 & 0.86 & 4.34 & 0.85 \\
\hline word2vec + Two-layer LSTM & 0.76 & 0.71 & 6.02 & 0.69 \\
\hline word2vec + Two-layer BLSTM & 0.78 & 0.83 & 4.79 & 0.82 \\
\hline word2vecpre-trained + Two-layer BLSTM & 0.79 & 0.91 & 3.2 & 0.92 \\
\hline SSWE + LSTM & 0.8 & 0.94 & 2.9 & 0.94 \\
\hline SSWE + BLSTM & 0.8 & 0.92 & 3.21 & 0.95 \\
\hline SSWE + Two-layer LSTM & 0.82 & 0.93 & 3 & 0.94 \\
\hline SSWE + Two-layer BLSTM & $\mathbf{0 . 9 1}$ & $\mathbf{0 . 9 6}$ & $\mathbf{2 . 4}$ & $\mathbf{0 . 9 6}$ \\
\hline
\end{tabular}

Table 1: Results of the different models on the Kaggle dataset. All resulting vectors were trained using linear regression. We optimized the parameters using a separate validation set (see text) and report the results on the test set.

Since the search space would be massive for grid search, the best hyperparameters were determined using Bayesian Optimization (Snoek et al., 2012). In this context, the performance of our models in the validation set is modeled as a sample from a Gaussian process (GP) by constructing a probabilistic model for the error function and then exploiting this model to make decisions about where to next evaluate the function. The hyperparameters for our baselines were also determined using the same methodology.

All models are trained on our training set (see Section 4), except the one prefixed 'word2 vec pre-trained' which uses pre-trained embeddings on the Google News Corpus. We report the Spearman's rank correlation coefficient $\rho$, Pearson's product-moment correlation coefficient $r$, and the root mean square error (RMSE) between the predicted scores and the gold standard on our test set, which are considered more appropriate metrics for evaluating essay scoring systems (Yannakoudakis and Cummins, 2015). However, we also report Cohen's $\kappa$ with quadratic weights, which was the evaluation metric used in the Kaggle competition. Performance of the models is shown in Table 1.

In terms of correlation, SVMs produce competitive results ( $\rho=0.78$ and $r=0.77$ ), outperforming doc2vec, LSTM and BLSTM, as well as their deep counterparts. As described above, the SVM model has rich linguistic knowledge and consists of hand-picked features which have achieved excellent performance in similar tasks (Yannakoudakis et al., 2011). However, in terms of RMSE, it is among the lowest performing models (8.85), together with 'BLSTM' and 'Twolayer BLSTM'. Deep models in combination with word2vec (i.e., 'word2vec + Two-layer LSTM' and 'word2vec + Two-layer BLSTM') and SVMs are comparable in terms of $r$ and $\rho$, though not in terms of RMSE, where the former produce better results, with RMSE improving by half (4.79). doc $2 \mathrm{vec}$ also produces competitive RMSE results (4.43), though correlation is much lower ( $\rho=0.62$ and $r=0.63$ ).

The two BLSTMs trained with word2vec embeddings are among the most competitive models in terms of correlation and outperform all the models, except the ones using pre-trained embeddings and SSWEs. Increasing the number of hidden layers and/or adding bi-directionality does not always improve performance, but it clearly helps in this case and performance improves compared to their uni-directional counterparts.

Using pre-trained word embeddings improves the results further. More specifically, we found 'word2vecpre-trained + Two-layer BLSTM' to be the best configuration, increasing correlation to $0.79 \rho$ and $0.91 r$, and reducing RMSE to 3.2. We note however that this is not an entirely 
fair comparison as these are trained on a much larger corpus than our training set (which we use to train our models). Nevertheless, when we use our SSWEs models we are able to outperform 'word2 vec pre-trained + Two-layer BLSTM', even though our embeddings are trained on fewer data points. More specifically, our best model ('SSWE + Two-layer BLSTM') improves correlation to $\rho=0.91$ and $r=0.96$, as well as RMSE to 2.4 , giving a maximum increase of around $10 \%$ in correlation. Given the results of the pre-trained model, we believe that the performance of our best SSWE model will further improve should more training data be given to it. ${ }^{7}$

\subsection{Discussion}

Our SSWE + LSTM approach having no prior knowledge of the grammar of the language or the domain of the text, is able to score the essays in a very human-like way, outperforming other stateof-the-art systems. Furthermore, while we tuned the models' hyperparameters on a separate validation set, we did not perform any further preprocessing of the text other than simple tokenization.

In the essay scoring literature, text length tends to be a strong predictor of the overall score. In order to investigate any possible effects of essay length, we also calculate the correlation between the gold scores and the length of the essays. We find that the correlations on the test set are relatively low $(r=0.3, \rho=0.44)$, and therefore conclude that there are no such strong effects.

As described above, we used Bayesian Optimization to find optimal hyperparameter configurations in fewer steps than in regular grid search. Using this approach, the optimization model showed some clear preferences for some parameters which were associated with better scoring models: ${ }^{8}$ the number of 'noisy' sequences $E$, the weighting factor $\alpha$ and the size of the LSTM layer $D_{L S T M}$. The optimal $\alpha$ value was consistently set to 0.1 , which shows that our SSWE approach was necessary to capture the usage of the words. Performance dropped considerably as $\alpha$ increased (less weight on SSWEs and more on the contextual aspect). When using $\alpha=1$, which

\footnotetext{
${ }^{7}$ Our approach outperforms all the other models in terms of Cohen's $\kappa$ too.

${ }^{8}$ For the best scoring model the hyperparameters were as follows: $D=200, H=100, \eta=1 e-7, n=9, E=$ $200, \alpha=0.1, D_{L S T M}=10, r=0.5$.
}

is equivalent to using the basic $\mathrm{C} \& \mathrm{~W}$ model, we found that performance was considerably lower (e.g., correlation dropped to $\rho=0.15$ ).

The number of 'noisy' sequences was set to 200 , which was the highest possible setting we considered, although this might be related more to the size of the corpus (see Mikolov et al. (2013) for a similar discussion) rather than to our approach. Finally, the optimal value for $D_{L S T M}$ was 10 (the lowest value investigated), which again may be corpus-dependent.

\section{Visualizing the black box}

In this section, inspired by recent advances in (de-) convolutional neural networks in computer vision (Simonyan et al., 2013) and text summarization (Denil et al., 2014), we introduce a novel method of generating interpretable visualizations of the network's performance. In the present context, this is particularly important as one advantage of the manual methods discussed in $\S 2$ is that we are able to know on what grounds the model made its decisions and which features are most discriminative.

At the outset, our goal is to assess the 'quality' of our word vectors. By 'quality' we mean the level to which a word appearing in a particular context would prove to be problematic for the network's prediction. In order to identify 'high' and 'low' quality vectors, we perform a single pass of an essay from left to right and let the LSTM make its score prediction. Normally, we would provide the gold scores and adjust the network weights based on the error gradients. Instead, we provide the network with a pseudo-score by taking the maximum score this specific essay can take ${ }^{9}$ and provide this as the 'gold' score. If the word vector is of 'high' quality (i.e., associated with higher scoring texts), then there is going to be little adjustment to the weights in order to predict the highest score possible. Conversely, providing the minimum possible score (here 0), we can assess how 'bad' our word vectors are. Vectors which require minimal adjustment to reach the lowest score are considered of 'lower' quality. Note that since we do a complete pass over the network (without doing any weight updates), the vector quality is going to be essay dependent.

\footnotetext{
${ }^{9}$ Note the in the Kaggle dataset essays from different essay sets have different maximum scores. Here we take as $\tilde{y}_{\max }$ the essay set maximum rather than the global maximum.
} 


\begin{tabular}{|c|}
\hline$\ldots$ way to show that Saeng is a determined $\ldots$ \\
\hline ... sometimes I do . Being patience is being ... \\
\hline ... which leaves the reader satisfied \\
\hline $\begin{array}{c}\ldots \text { is in this picture the cyclist is riding a dry and area which could mean that it is very } \\
\text { and the looks to be going down hill there looks to be a lot of turns } \ldots\end{array}$ \\
\hline $\begin{array}{c}\ldots \text { The only reason im putting this in my own way is because know one is } \\
\text { patient in my family .... }\end{array}$ \\
\hline $\begin{array}{l}\text {... Whether they are building hand-eye coordination , researching a country , or family and } \\
\text { friends through @CAPS } 3 \text {, @CAPS2, @ CAPS6 the internet is highly and } \\
\text { I hope you feel the same way. }\end{array}$ \\
\hline
\end{tabular}

Table 2: Several example visualizations created by our LSTM. The full text of the essay is shown in black and the 'quality' of the word vectors appears in color on a range from dark red (low quality) to dark green (high quality).

Concretely, using the network function $f(\mathbf{x})$ as computed by Eq. (12) - (17), we can approximate the loss induced by feeding the pseudo-scores by taking the magnitude of each error vector (18) (19). Since $\lim _{\|\mathbf{w}\|_{2} \rightarrow 0} \hat{y}=y$, this magnitude should tell us how much an embedding needs to change in order to achieve the gold score (here pseudo-score). In the case where we provide the minimum as a pseudo-score, a $\|\mathrm{w}\|_{2}$ value closer to zero would indicate an incorrectly used word. For the results reported here, we combine the magnitudes produced from giving the maximum and minimum pseudo-scores into a single score, computed as $L\left(\tilde{y}_{\max }, f(\mathbf{x})\right)-L\left(\tilde{y}_{\min }, f(\mathbf{x})\right)$, where:

$$
\begin{aligned}
L(\tilde{y}, f(\mathbf{x})) & \approx\|\mathbf{w}\|_{2} \\
\mathbf{w} & =\left.\nabla L(\mathbf{x}) \triangleq \frac{\partial L}{\partial \mathbf{x}}\right|_{(\tilde{y}, f(\mathbf{x}))}
\end{aligned}
$$

where $\|\mathbf{w}\|_{2}$ is the vector Euclidean norm $\mathbf{w}=$ $\sqrt{\sum_{i=1}^{N} w_{i}^{2}} ; L(\cdot)$ is the mean squared error as in Eq. (8); and $\tilde{y}$ is the essay pseudo-score.

We show some examples of this visualization procedure in Table 2. The model is capable of providing positive feedback. Correctly placed punctuation or long-distance dependencies (as in Sentence 6 are ...researching) are particularly favoured by the model. Conversely, the model does not deal well with proper names, but is able to cope with POS mistakes (e.g., Being patience or the internet is highly and ...). However, as seen in Sentence 3 the model is not perfect and returns a false negative in the case of satisfied.

One potential drawback of this approach is that the gradients are calculated only after the end of the essay. This means that if a word appears mul- tiple times within an essay, sometimes correctly and sometimes incorrectly, the model would not be able to distinguish between them. Two possible solutions to this problem are to either provide the gold score at each timestep which results into a very computationally expensive endeavour, or to feed sentences or phrases of smaller size for which the scoring would be more consistent. ${ }^{10}$

\section{Conclusion}

In this paper, we introduced a deep neural network model capable of representing both local contextual and usage information as encapsulated by essay scoring. This model yields score-specific word embeddings used later by a recurrent neural network in order to form essay representations.

We have shown that this kind of architecture is able to surpass similar state-of-the-art systems, as well as systems based on manual feature engineering which have achieved results close to the upper bound in past work. We also introduced a novel way of exploring the basis of the network's internal scoring criteria, and showed that such models are interpretable and can be further exploited to provide useful feedback to the author.

\section{Acknowledgments}

The first author is supported by the Onassis Foundation. We would like to thank the three anonymous reviewers for their valuable feedback.

\footnotetext{
${ }^{10}$ We note that the same visualization technique can be used to show the 'goodness' of phrases/sentences. Within the phrase setting, after feeding the last word of the phrase to the network, the LSTM layer will contain the phrase embedding. Then, we can assess the 'goodness' of this embedding by evaluating the error gradients after predicting the highest/lowest score.
} 


\section{References}

Yigal Attali and Jill Burstein. 2006. Automated essay scoring with e-Rater v.2.0. Journal of Technology, Learning, and Assessment, 4(3):1-30.

Ted Briscoe, John Carroll, and Rebecca Watson. 2006. The second release of the RASP system. In Proceedings of the COLING/ACL, volume 6.

Ted Briscoe, Ben Medlock, and Øistein E. Andersen. 2010. Automated assessment of ESOL free text examinations. Technical Report UCAM-CL-TR790, University of Cambridge, Computer Laboratory, nov.

Ciprian Chelba, Tomáš Mikolov, Mike Schuster, Qi Ge, Thorsten Brants, Phillipp Koehn, and Tony Robinson. 2013. One Billion Word Benchmark for Measuring Progress in Statistical Language Modeling. In arXiv preprint.

YY Chen, CL Liu, TH Chang, and CH Lee. 2010. An Unsupervised Automated Essay Scoring System. IEEE Intelligent Systems, pages 61-67.

Ronan Collobert and Jason Weston. 2008. A unified architecture for natural language processing: deep neural networks with multitask learning. Proceedings of the Twenty-Fifth international conference on Machine Learning, pages 160-167, July.

Ronan Collobert, Jason Weston, Leon Bottou, Michael Karlen, Koray Kavukcuoglu, and Pavel Kuksa. 2011. Natural language processing (almost) from scratch. Mar.

Scott Crossley, Laura K Allen, Erica L Snow, and Danielle S McNamara. 2015. Pssst... textual features... there is more to automatic essay scoring than just you! In Proceedings of the Fifth International Conference on Learning Analytics And Knowledge, pages 203-207. ACM.

Yann N. Dauphin, Harm de Vries, and Yoshua Bengio. 2015. Equilibrated adaptive learning rates for nonconvex optimization. Feb.

Misha Denil, Alban Demiraj, Nal Kalchbrenner, Phil Blunsom, and Nando de Freitas. 2014. Modelling, visualising and summarising documents with a single convolutional neural network. Jun.

Semire Dikli. 2006. An overview of automated scoring of essays. Journal of Technology, Learning, and Assessment, 5(1).

S. Elliot. 2003. Intellimetric ${ }^{\mathrm{TM}}$ : From here to validity. In M. D. Shermis and J. Burnstein, editors, $A u$ tomated Essay Scoring: A Cross-Disciplinary Perspective, pages 71-86. Lawrence Erlbaum Associates.

Noura Farra, Swapna Somasundaran, and Jill Burstein. 2015. Scoring persuasive essays using opinions and their targets. In Proceedings of the Tenth Workshop on Innovative Use of NLP for Building Educational Applications, pages 64-74.
Alex Graves. 2012. Supervised Sequence Labelling with Recurrent Neural Networks. Springer Berlin Heidelberg.

Michael U. Gutmann and Aapo Hyvärinen. 2012. Noise-contrastive estimation of unnormalized statistical models, with applications to natural image statistics. J. Mach. Learn. Res., 13:307-361, February.

Karl Moritz Hermann, Tom Koisk, Edward Grefenstette, Lasse Espeholt, Will Kay, Mustafa Suleyman, and Phil Blunsom. 2015. Teaching machines to read and comprehend. Jun.

S Hochreiter and J Schmidhuber. 1997. Long shortterm memory. Neural computation, 9(8):17351780.

Beata Beigman Klebanov and Michael Flor. 2013. Word association profiles and their use for automated scoring of essays. In Proceedings of the 51st Annual Meeting of the Association for Computational Linguistics, pages 1148-1158.

Thomas K. Landauer, Darrell Laham, and Peter W. Foltz. 2003. Automated scoring and annotation of essays with the Intelligent Essay Assessor. In M.D. Shermis and J.C. Burstein, editors, Automated essay scoring: A cross-disciplinary perspective, pages 87112.

Quoc V. Le and Tomas Mikolov. 2014. Distributed representations of sentences and documents. May.

Honglak Lee, Roger Grosse, Rajesh Ranganath, and Andrew Y. Ng. 2009. Convolutional deep belief networks for scalable unsupervised learning of hierarchical representations. Proceedings of the 26th Annual International Conference on Machine Learning ICML 09.

Deryle Lonsdale and D. Strong-Krause. 2003. Automated rating of ESL essays. In Proceedings of the HLT-NAACL 2003 Workshop: Building Educational Applications Using Natural Language Processing.

Danielle S McNamara, Scott A Crossley, Rod D Roscoe, Laura K Allen, and Jianmin Dai. 2015. A hierarchical classification approach to automated essay scoring. Assessing Writing, 23:35-59.

Tomáš Mikolov, Stefan Kombrink, Anoop Deoras, Lukáš Burget, and Jan Černocký. 2011. RNNLM-Recurrent neural network language modeling toolkit. In ASRU 2011 Demo Session.

Tomas Mikolov, I Sutskever, K Chen, G S Corrado, and Jeffrey Dean. 2013. Distributed representations of words and phrases and their compositionality. In Advances in Neural Information Processing Systems, pages 3111-3119.

Ellis B. Page. 1967. Grading essays by computer: progress report. In Proceedings of the Invitational Conference on Testing Problems, pages 87-100. 
Ellis B. Page. 1968. The use of the computer in analyzing student essays. International Review of Education, 14(2):210-225, June.

E.B. Page. 2003. Project essay grade: PEG. In M.D. Shermis and J.C. Burstein, editors, Automated essay scoring: A cross-disciplinary perspective, pages 4354.

L.M. Rudner and Tahung Liang. 2002. Automated essay scoring using Bayes' theorem. The Journal of Technology, Learning and Assessment, 1(2):3-21.

Keisuke Sakaguchi, Michael Heilman, and Nitin Madnani. 2015. Effective feature integration for automated short answer scoring. In Proceedings of the Tenth Workshop on Innovative Use of NLP for Building Educational Applications.

M Shermis and B Hammer. 2012. Contrasting stateof-the-art automated scoring of essays: analysis. Technical report, The University of Akron and Kaggle.

Mark D Shermis. 2015. Contrasting state-of-the-art in the machine scoring of short-form constructed responses. Educational Assessment, 20(1):46-65.

Karen Simonyan, Andrea Vedaldi, and Andrew Zisserman. 2013. Deep inside convolutional networks: Visualising image classification models and saliency maps. 12.

D.D.K. Sleator and D. Templerley. 1995. Parsing English with a link grammar. Proceedings of the 3rd International Workshop on Parsing Technologies, ACL.

Jasper Snoek, Hugo Larochelle, and Ryan P. Adams. 2012. Practical bayesian optimization of machine learning algorithms. Jun.

Swapna Somasundaran, Jill Burstein, and Martin Chodorow. 2014. Lexical chaining for measuring discourse coherence quality in test-taker essays. In COLING, pages 950-961.

Ilya Sutskever, Oriol Vinyals, and Quoc V. Le. 2014. Sequence to sequence learning with neural networks. Sep.

Kai Sheng Tai, Richard Socher, and Christopher D. Manning. 2015. Improved semantic representations from tree-structured long short-term memory networks. Feb.

Duyu Tang. 2015. Sentiment-specific representation learning for document-level sentiment analysis. In Proceedings of the Eighth ACM International Conference on Web Search and Data Mining - WSDM '15. Association for Computing Machinery (ACM).

D. M. Williamson. 2009. A framework for implementing automated scoring. Technical report, Educational Testing Service.
Helen Yannakoudakis and Ronan Cummins. 2015. Evaluating the performance of automated text scoring systems. In Proceedings of the Tenth Workshop on Innovative Use of NLP for Building Educational Applications. Association for Computational Linguistics (ACL).

Helen Yannakoudakis, Ted Briscoe, and Ben Medlock. 2011. A new dataset and method for automatically grading ESOL texts. In The 49th Annual Meeting of the Association for Computational Linguistics: Human Language Technologies, Proceedings of the Conference, 19-24 June, 2011, Portland, Oregon, USA, pages 180-189. 